\title{
Long non-coding RNA SNHG8 enhances triple-negative breast cancer cell proliferation and migration by regulating the miR-335-5p/PYGO2 axis
}

\author{
Jintao Qian, Xinhan Lei, Yue Sun, Lu Zheng, Jia Li, Shuai Zhang, Lei Zhang, Wanwan Li, Jianing Shi,
} Wenjun Jia* and Tong Tang*

\begin{abstract}
Background: Growing evidence has demonstrated that long non-coding RNAs (IncRNAs) can function as modulators in the development of triple-negative breast cancer (TNBC). However, the function of IncRNA small nucleolar RNA host gene 8 (SNHG8) in TNBC remains unclear. Therefore, our study aimed at investigating the role of SNHG8 in the proliferation and migration of TNBC cells.

Methods: SNHG8 expression was evaluated using RT-qPCR assay. Cell proliferation and migration were assessed by EdU, colony formation and Transwell assays. The levels of proteins related to EMT process were examined by western blot assay. The interaction among SNHG8, miR-335-5p and pygopus family PHD finger 2 (PYGO2) was detected by RIP assay, RNA pull down assay and luciferase reporter assay.

Results: SNHG8 expression was significantly up-regulated in TNBC cells. SNHG8 silencing obviously inhibited TNBC cell proliferation, migration and EMT process. Moreover, SNHG8 acted as a sponge to sequester miR-335-5p in TNBC cells. Besides, PYGO2 was proven as a target gene of miR-335-5p, and SNHG8 promoted TNBC cell proliferation, migration and EMT process through regulating miR-335-5p and PYGO2.

Conclusions: Totally, our study indicated that SNHG8 promoted TNBC cell proliferation and migration by regulating the miR-335-5p/PYGO2 axis.
\end{abstract}

Keywords: Triple-negative breast cancer, SNHG8, miR-335-5p, PYGO2

\section{Background}

Breast cancer is one of the most common cancers in female worldwide [1]. The incidence of breast cancer occurs $25.1 \%$ of all cancers, and the mortality accounts for approximately $6.6 \%$ of global cancer death rate, making it the second cause of cancer-related death for women $[2,3]$. Triple-negative breast cancer (TNBC) is a subtype of breast cancer and characterized by negative estrogen,

\footnotetext{
*Correspondence: jiawenjun@smail.nju.edu.cn; tt20164@126.com Department of General Surgery, the Second Affiliated Hospital of Anhui Medical University, Hefei 230601, Anhui, China
}

progesterone receptors and proto-oncogene HER2 [4]. On account of high metastatic risk and lack of targeted therapy, the prognosis of patients with TNBC is poor [5]. As a consequence, a better understanding of the underlying mechanisms of TNBC progression may be conducive to the development of more novel and effective therapeutic strategies.

Long non-coding RNAs (lncRNAs) are generally classified as a category of RNAs longer than 200 nucleotides and cannot encode proteins [6,7]. LncRNAs are involved in many biological processes ranging from

(c) The Author(s). 2021 Open Access This article is licensed under a Creative Commons Attribution 4.0 International License, which permits use, sharing, adaptation, distribution and reproduction in any medium or format, as long as you give appropriate credit to the original author(s) and the source, provide a link to the Creative Commons licence, and indicate if changes were made. The images or other third party material in this article are included in the article's Creative Commons licence, unless indicated otherwise in a credit line to the material. If material is not included in the article's Creative Commons licence and your intended use is not permitted by statutory regulation or exceeds the permitted use, you will need to obtain permission directly from the copyright holder. To view a copy of this licence, visit http://creativecommons.org/licenses/by/4.0/ The Creative Commons Public Domain Dedication waiver (http://creativecommons.org/publicdomain/zero/1.0/) applies to the data made available in this article, unless otherwise stated in a credit line to the data. 
housekeeping functions including transcription to more specific functions including genomic imprinting [8]. Of note, lncRNAs are also involved in a variety of activities such as the degradation and translation of messenger RNA or serving as RNA decoys or scaffolds [9]. Increasing evidences have demonstrated that the abnormal expression of lncRNAs can function as oncogenes or tumor suppressors to participate in the occurrence and development of cancers, including TNBC. For instance, overexpressed lncRNA GAS5 facilitates chemosensitivity and apoptosis of TNBC cells [10]. WT1-AS is with low expression in TNBC cells and exerts anticancer function in cell migration and invasion [11]. Moreover, some lncRNAs can function as potential prognostic biomarkers for TNBC [12]. LncRNA small nucleolar RNA host gene 8 (SNHG8) has been confirmed to exert carcinogenic function in many types of tumors. For instance, SNHG8 accelerates cell growth through sponging miR-663 in colorectal cancer cells [13]. ,SNHG8 promotes hepatocellular cancer tumorigenesis by sponging miR-149-5p [14]. SNHG8 acts as an oncogene of nonsmall-cell lung cancer via miR-542-3p to regulate CCND1/CDK6 [15]. However, the molecule mechanism and function of SNHG8 in TNBC remained unclear.

Therefore, the aim of our research was to investigate the function and detailed mechanism of SNHG8 in TNBC. We probed the expression of SNHG8 in TNBC and investigated the impacts of SNHG8 on TNBC cell proliferation, migration and epithelial-mesenchymal transition (EMT) process. Cytoplasmic lncRNAs are known as competing endogenous RNA (ceRNA) through sponging miRNAs in human cancers [16-18]. Here, we also explored whether SNHG8 played a similar role in TNBC.

Collectively, our study explored the function of SNHG8 and its downstream molecular mechanism in TNBC.

\section{Results \\ SNHG8 facilitates the proliferation, migration and EMT process of TNBC cells}

To basically understand the function of SNHG8 on the biological behaviors of TNBC cells, its expression in different cell lines was detected. We firstly evaluated SNHG8 expression in four common TNBC cell lines (MDA-MB-231, MDA-MB-436, BT-549 and HCC1937) compared with that in normal breast epithelial cells (MCF-10A). The result of RT-qPCR analysis indicated that SNHG8 expression was higher in each TNBC cell line than that in normal cells (Fig. 1A). To further explore whether SNHG8 influenced TNBC cell behaviors, the interference efficiency of SNHG8 was detected in MDA-MB-231 and BT-549 (which exhibited higher SNHG8 levels than other TNBC cells) through transfecting three shRNAs targeting SNHG8, respectively (Fig. 1B). According to the result of RT-qPCR assay, no significant difference was observed among the interference efficiency of sh/SNHG8\#1, sh/SNHG8\#2 and sh/SNHG8\#3. Therefore, we selected sh/SNHG8\#1 and sh/SNHG8\#2 for the following assays. After that, the influence of SNHG8 silencing on cell growth and proliferation was inspected by colony formation assay and EdU assay. As shown by the results, the number of colonies was decreased by down-regulated SNHG8 (Fig. 1C). Similarly, SNHG8 silencing obviously inhibited the proliferative ability of TNBC cells (Fig. 1D). Besides, through Transwell assay, we observed that SNHG8 silencing impeded cell migratory capacity (Fig. 1E). Furthermore, as shown in Fig. 1F, SNHG8 knockdown increased E-cadherin mRNA and protein levels, while Ncadherin, Vimentin, MMP2 and MMP7 were downregulated, suggesting that EMT process was repressed by SNHG8 depletion. Totally, SNHG8 played an oncogenic role in TNBC cells through facilitating the cell proliferation, migration and EMT process.

\section{SNHG8 serves as a sponge for miR-335-5p}

To investigate the regulatory mechanism of SNHG8 in TNBC cells, subcellular separation assay and FISH assay were performed. As shown by the figures, it was exhibited that the major distribution of SNHG8 was in the cytoplasm (Fig. 2A and B). Moreover, by using ENCORI online bioinformatic tool (http://starbase.sysu.edu.cn), it was predicted that miR-335-5p could interact with SNHG8 under specific screening condition (DegradomeData $\geq 1$ ). Then, we conducted RIP assay in TNBC cells and found that both SNHG8 and miR-335-5p were highly enriched in Anti-Ago2 groups rather than in Anti-IgG groups, which indicated that they could coexist in TNBC cells (Fig. 2C). Taken together, SNHG8 was able to sequester miR-335-5p in TNBC cells.

\section{PYGO2 is a target gene of miR-335-5p}

To determine the possible downstream genes of miR335-5p, we utilized ENCORI and sifted out six candidate genes (VAPA, ATP1B1, FAM107B, PYGO2, RPE and ARHGAP18) which possessed possible binding ability to miR-335-5p under specific screening condition (Degradome-Data $\geq 2$ ) according to TargetScan targetpredicting program. Besides, according to the results of RT-qPCR, it was suggested that only PYGO2 was downregulated in TNBC cells transfected with miR-335-5p mimics, thus we selected PYGO2 for further experiments (Fig. 3A). Then, RT-qPCR assay was carried out and it was demonstrated that PYGO2 expression was markedly elevated in several TNBC cells compared with that in MCF-10A cell line (Fig. 3B). Furthermore, according to the result of RIP assay, SNHG8, miR-335-5p 


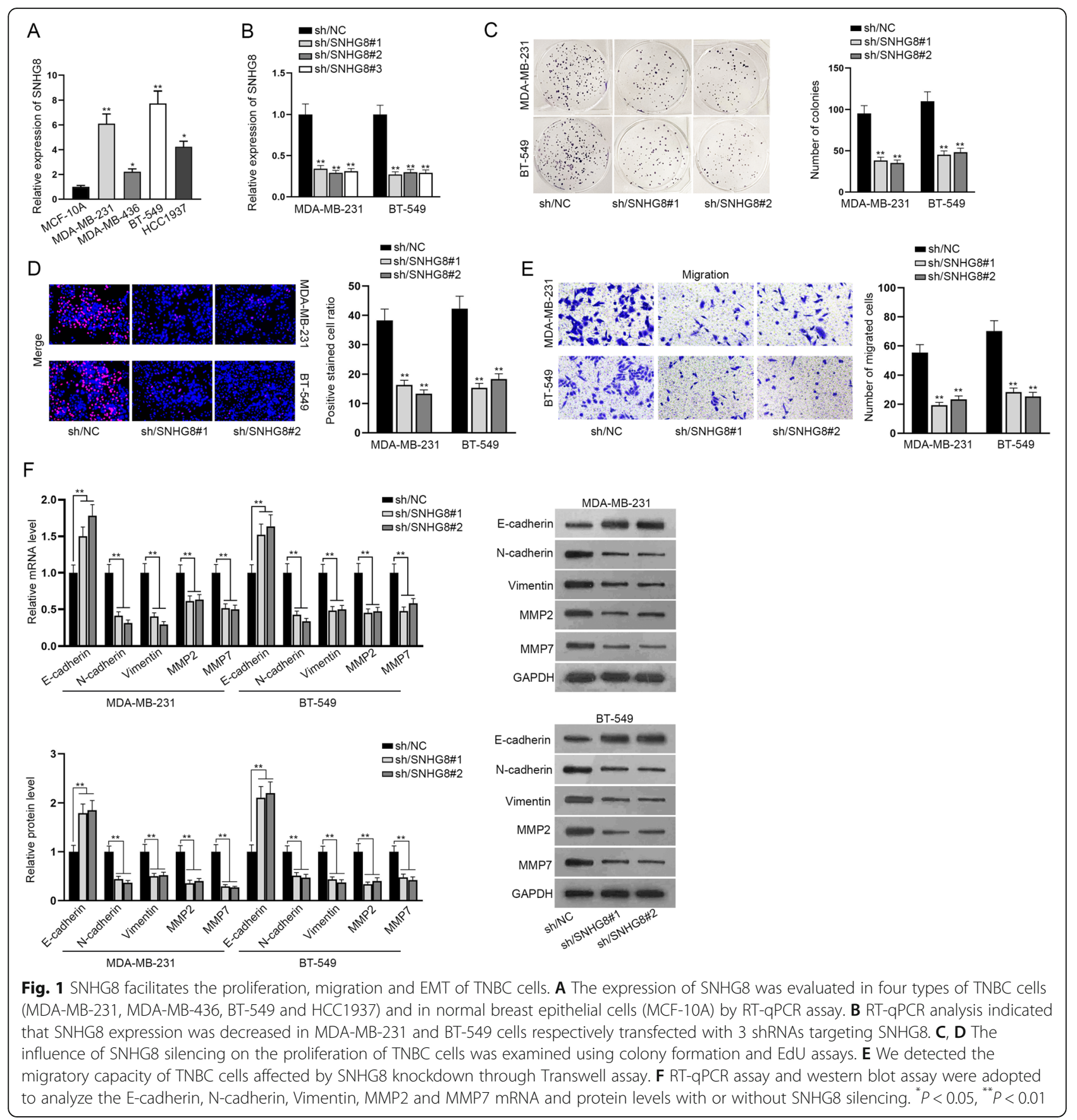

and PYGO2 were significantly enriched in Anti-Ago2 groups, indicating that those three RNAs co-existed in RISCs (Fig. 3C). Moreover, by utilizing the RNA pull down assay, it was found that both SNHG8 and PYGO2 were obviously enriched in Bio-miR-335-5pWT groups (Fig. 3D). Hence, it was certified that miR-335-5p could bind to both SNHG8 and PYGO2. Meanwhile, in MDA-MB-231, BT549 as well as HEK$293 \mathrm{~T}$ cells, luciferase reporter assay further demonstrated that miR-335-5p overexpression markedly reduced the luciferase activity of pmirGLO/SNHG8WT or pmirGLO/3'UTR-WT, but there were no conspicuous changes in response to miR-335-5p mimics in mutant groups or normal control groups (Fig. 3E and F). Finally, we found that the luciferase activity of the PYGO2 3'UTR-WT group, which had been reduced after the overexpression of miR-335-5p, was partially rescued after SNHG8 up-regulation (Fig. 3G). Altogether, we found that in TNBC cells, PYGO2 was a target gene of miR-335-5p. 


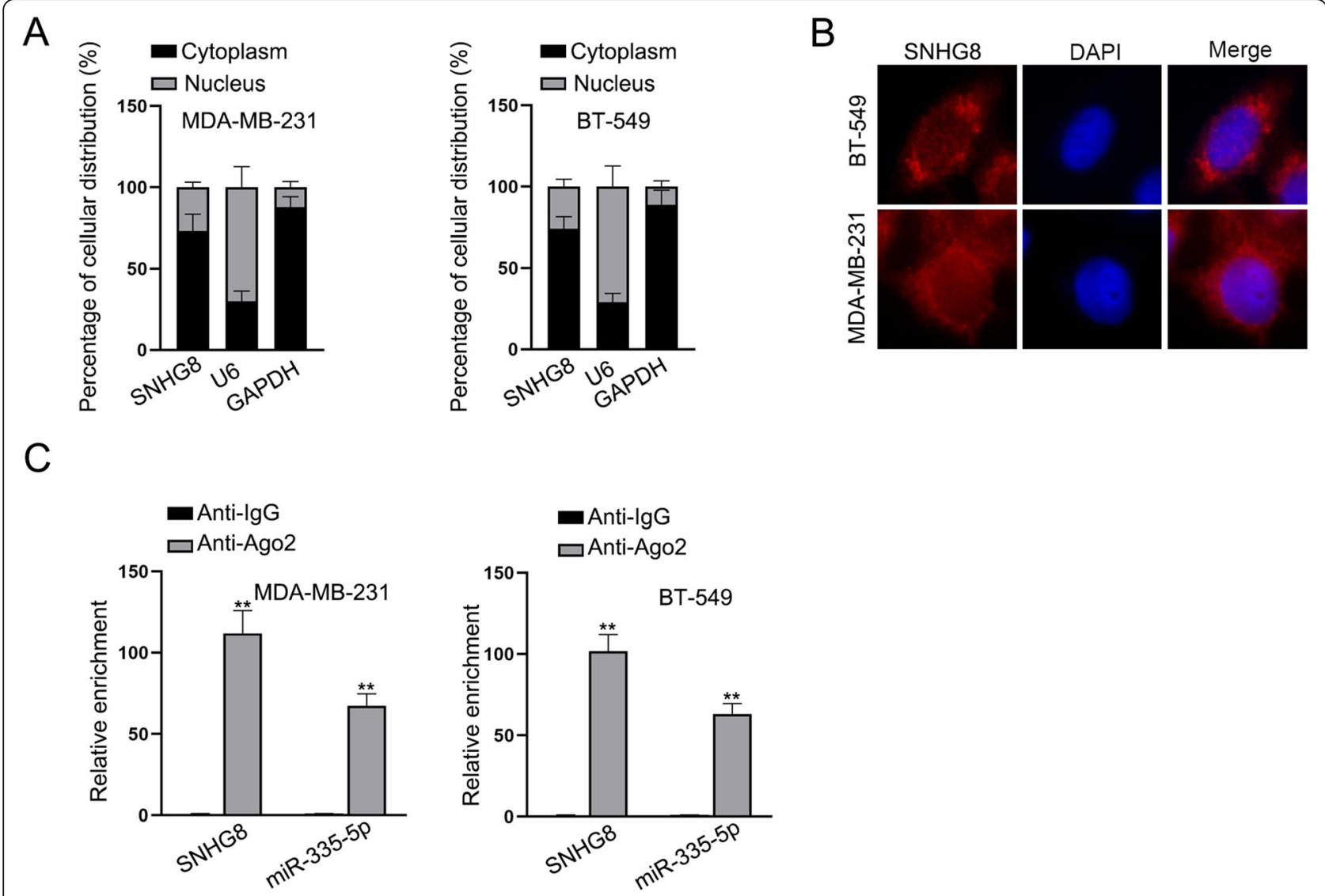

Fig. 2 SNHG8 serves as a sponge for miR-335-5p. A, B Subcellular separation assay and FISH assay were conducted to confirm the distribution of SNHG8. C RIP experiment was conducted and it was verified that SNHG8 and miR-335-5p were enriched in the groups of Anti-Ago2. ${ }^{* *} P<0.01$

SNHG8 promotes the proliferation, migration and EMT process of TNBC cells by sponging miR-335-5p

Further, several rescue experiments were conducted to explore the relationship between miR-335-5p and SNHG8. First of all, through RT-qPCR assay, the interference efficiency of miR-335-5p was detected and a satisfactory result was observed (Fig. 4A). After that, through colony formation assay, we observed that TNBC cell proliferation reduced by SNHG8 silencing was enhanced after the co-transfection of miR-335-5p inhibitor (Fig. 4B). In addition, it was revealed in Transwell assay that SNHG8 knockdown reduced the number of migrated cells, while miR-335-5p depletion could partially reverse the suppressive effect (Fig. 4C). In addition, Ecadherin mRNA and protein levels were up-regulated by SNHG8 knockdown, and such result was partially reversed by miR-335-5p down-regulation. However, the levels of N-cadherin, Vimentin, MMP2 as well as MMP7 exhibited a contrary tendency (Fig. 4D). Totally, miR335-5p was sponged by SNHG8, thus enhancing the proliferation, migration and EMT process of TNBC cells.
The tumor-suppressive role of SNHG8 silencing on the proliferation, migration and EMT process of TNBC cells were reversed by PYGO2

Subsequently, we investigated the relationship between SNHG8 and PYGO2 in TNBC cells. After the transfection of pcDNA3.1-PYGO2 into TNBC cells, the overexpression efficiency of PYGO2 was detected and a satisfactory result was observed (Fig. 5A). In colony formation assay, SNHG8 silencing reduced the proliferative ability of TNBC cells, while the co-transfection of pcDNA3.1-PYGO2 could partially rescue the inhibitory effect (Fig. 5B). According to the result of Transwell assay, TNBC cell migration was weakened by SNHG8 down-regulation but the co-transfection of pcDNA3.1PYGO2 could counteract the falling trend of migration (Fig. 5C). Through RT-qPCR and western blot assays, we found that E-cadherin mRNA and protein levels were increased by SNHG8 silencing but reduced after the cotransfection of pcDNA3.1-PYGO2. Besides, those of Ncadherin, Vimentin, MMP2 as well as MMP7 were suppressed in response to SNHG8 knockdown, but was partially reversed after the co-transfection of pcDNA3.1- 


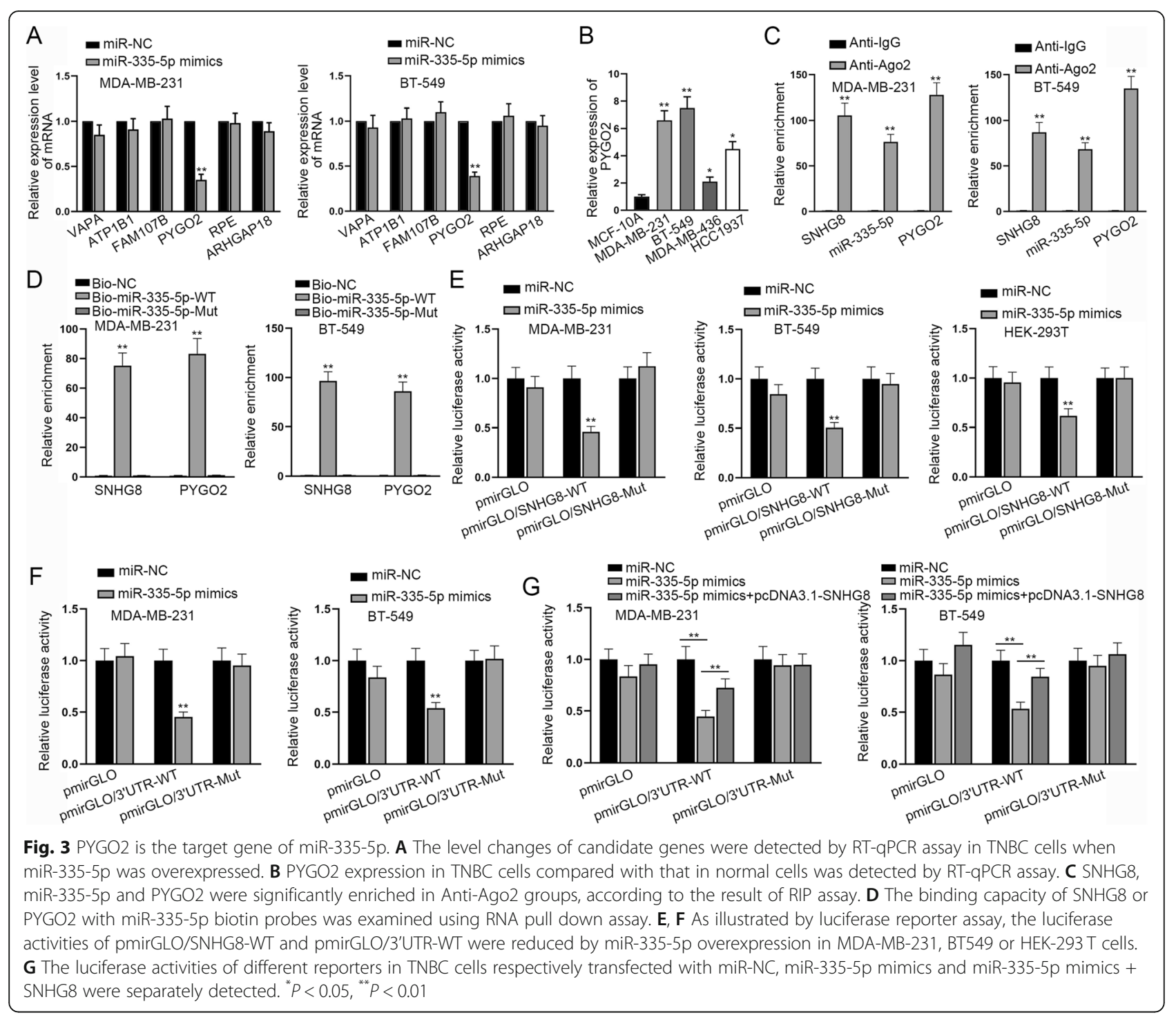

PYGO2 (Fig. 5D). Together, those results indicated that SNHG8 contributed to TNBC cell proliferation, migration and EMT via regulating PYGO2.

\section{Discussion}

A large number of studies have proven that the aberrant expression of lncRNAs is closely related to the progression of cancers. SNHG8 is up-regulated in pancreatic adenocarcinoma and the pancreatic adenocarcinoma patients with higher expression of SHNG8 are accompanied with shorter overall survival [19]. In addition, SNHG8 is highly expressed in esophageal squamous cell carcinoma and high SNHG8 expression is closely associated with TNM stage and worse overall survival among patients with esophageal squamous cell carcinoma [20]. Nevertheless, the role of SNHG8 in TNBC remains unclear. In our study, we found that SNHG8 expression was significantly elevated in TNBC cells, which suggested the oncogenic potential of SNHG8 in TNBC.

As reported previously, SNHG8 exerts oncogenic functions in the progression of many cancers. In gastric cancer, SNHG8 enhances cell proliferation and invasion [21]. In endometrial carcinoma, the knockdown of SNHG8 represses cell viability [22]. In colorectal cancer, SNHG8 accelerates the growth, migration and invasion of cells [13]. In line with those studies, our research also proved that SNHG8 deletion significantly impeded cell proliferation, migration and the EMT process of TNBC. All above results revealed the important role of SNHG8 in the malignant behaviors of TNBC, mirroring that SNHG8 might be a novel target for TNBC treatment.

Moreover, our study further found that SNHG8 could function as a ceRNA to exert functions at posttranscriptional level in TNBC cells. According to recent 


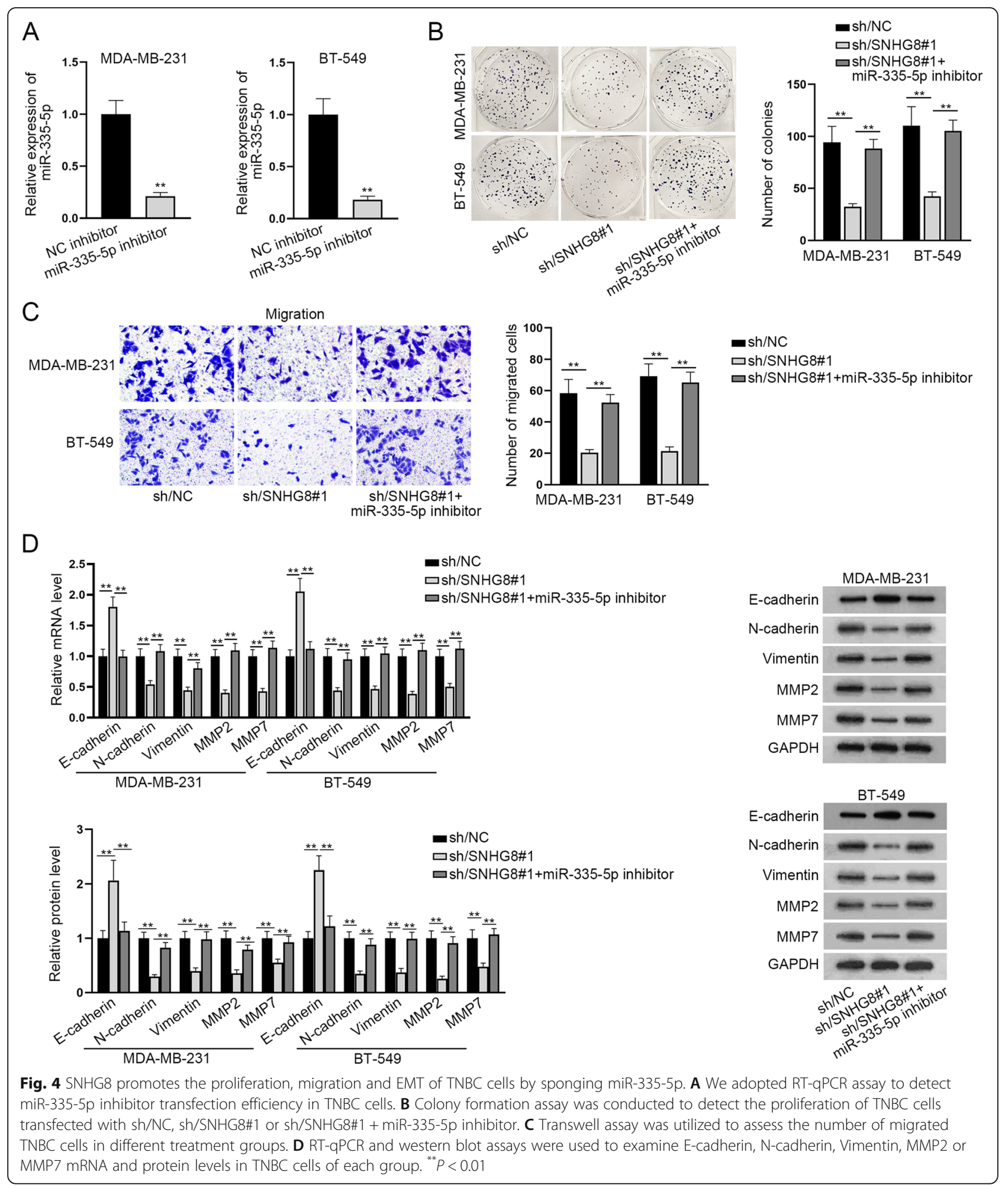

studies, SNHG8 contributes to the cell proliferation of osteosarcoma via binding to miR-542-3p [23]. Moreover, SNHG8 acts as a sponge of miR-149 to regulate the tumorigenesis and metastasis of hepatocellular carcinoma [14]. Consistently, our study further discovered that
SNHG8 combined with miR-335-5p in TNBC cells. Moreover, rescue experiments confirmed that miR-335$5 p$ inhibitor could partially reverse the inhibited effects of SNHG8 silence on TNBC proliferation, migration and the EMT process. 


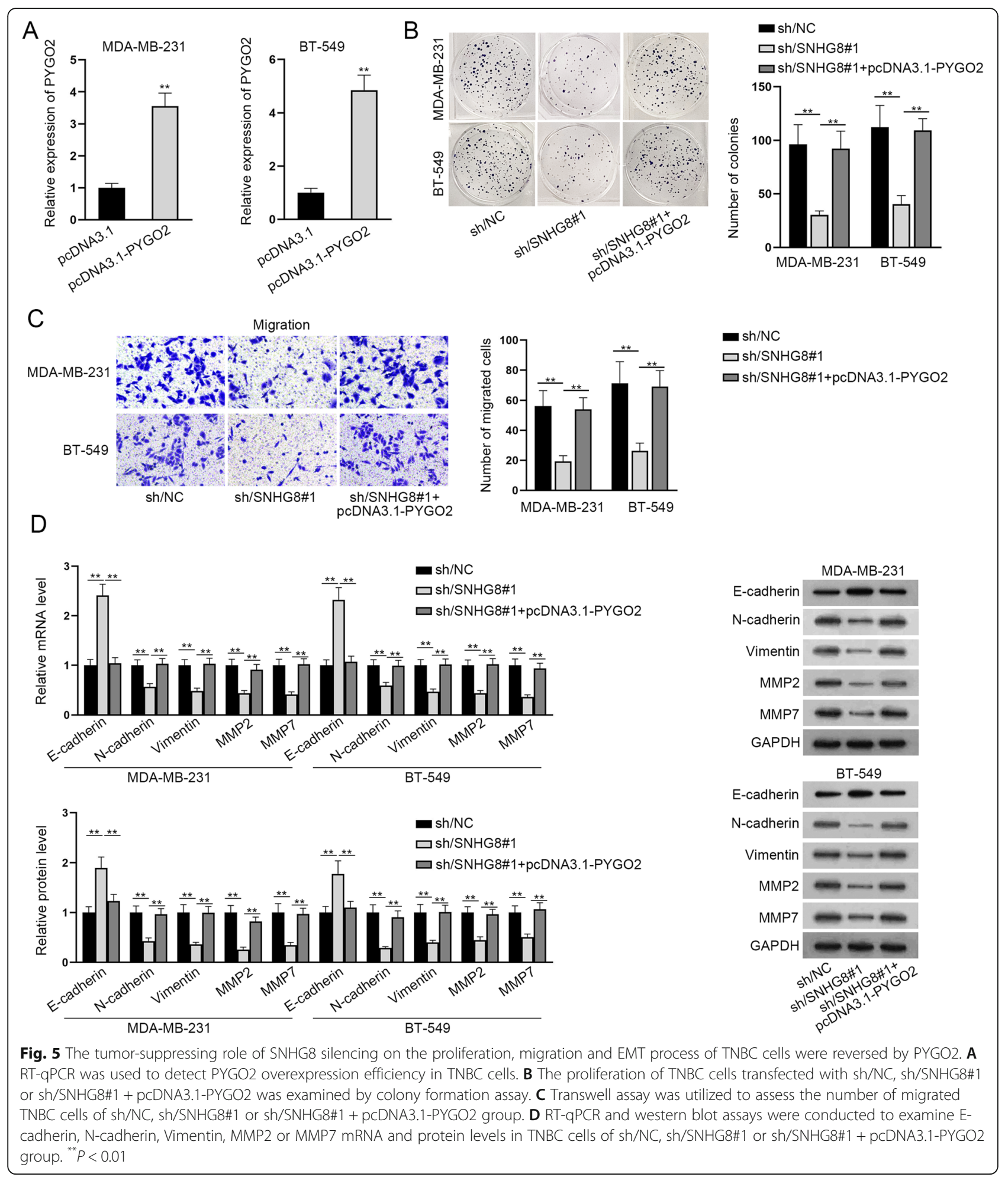

It is well documented that miRNAs function posttranscriptionally by usually base-pairing to the mRNAs 3 '-untranslated regions to suppress protein synthesis [24]. It has been reported that miR-335-5p can inhibit thyroid cancer cells invasion and metastasis via targeting
ICAM-1 [25]. In addition, miR-335-5p weakens colorectal cancer cell proliferation and migration via reducing LDHB [26]. In our study, we found that PYGO2 was the target gene of miR-335-5p in TNBC cells. Notably, PYGO2 has been proven to be up-regulated in breast 
cancer [27]. Here, we found that PYGO2 was upregulated in TNBC cells and recovered SNHG8mediated cellular function.

\section{Conclusion}

In conclusion, our current study was the first in the field to reveal the role of SNHG8/miR-335-5p/PYGO2 pathway in TNBC cell proliferation, migration and EMT process. However, the lack of investigation on the upstream target of SNHG8 is a limitation of our current study. Therefore, we will further explore the upstream molecular mechanism of SNHG8 in our future study.

\section{Materials and methods Cell culture}

Human normal breast epithelial cell line (MCF-10A), human TNBC cell lines (MDA-MB-231, MDA-MB-436, BT-549, HCC1937) as well as human embryonic kidney cell line (HEK-293 T) were all procured from the American Type Culture Collection (ATCC; Manassas, VA, USA) and preserved at $37{ }^{\circ} \mathrm{C}$ with $5 \% \mathrm{CO}_{2}$. Leibovitz's L15 medium (Thomas Scientific, Swedesboro, NJ, USA) was applied to incubate MDA-MB-231 and MDA-MB436 cells; ATCC-formulated RPMI-1640 medium (ATCC) was used for cultivating BT-549 and HCC1937 cells; Mammary Epithelial Cell Growth Medium (MEGM; Gibco, Grand Island, NY, USA) was adopted for cultivating MCF-10A cells; Dulbecco's modified Eagle medium (DMEM; Gibco) was used for incubating HEK-293 T cells. 10\% fetal bovine serum (FBS; Gibco) together with $1 \%$ penicillin-streptomycin (Sigma-Aldrich, St. Louis, MO, USA) was also applied to all media.

\section{Real-time quantitative polymerase chain reaction (RT- qPCR)}

Total RNA in cultured cells was first extracted by using TRIzol Reagent (Invitrogen, Carlsbad, CA, USA). PrimeScript $^{\mathrm{tm}}$ II Reverse Transcriptase (Takara, Kusatsu, Japan) was used for complementary DNA (cDNA) synthesis. CFX96 Touch Real-Time PCR Detection System (BioRad, Hercules, CA, USA) together with SYBR Green PCR Kit (Takara) was applied to conduct PCR. All results were calculated by $2^{-\Delta \Delta \mathrm{Ct}}$ method. The experiment was conducted for three times in an independent manner.

\section{Cell transfection}

Confluent TNBC cells at $80-90 \%$ confluence were seeded into 6 -well plates at $1 \times 10^{6}$ cells/well for 48 -h transfection with the specific short hairpin RNAs (shRNAs; GenePharma, Shanghai, China) against SNHG8 (sh/SNHG8\#1 and \#2), and nonspecific shRNAs (sh/NC) were used in negative control (NC) group. Besides, miR-335-5p mimics/inhibitor together with their relative NCs (miR-NC) were procured form RiboBio (Guangzhou, China). Overexpression of SNHG8 or PYGO2 was achieved by recombinant plasmids pcDNA3.1-SNHG8 or pcDNA3.1-PYGO2, and empty vectors were used as NC. Lipofectamine 3000 reagent (Invitrogen) was applied for cell transfection for $48 \mathrm{~h}$.

\section{Colony formation}

TNBC cells after transfection were seeded into 6-well cell culture plates (500 cells/well) and cultured for 14 days of colony formation. After that, colonies were immobilized by $4 \%$ paraformaldehyde, dyed by $0.5 \%$ crystal violet for $20 \mathrm{~min}$ and counted manually. The experiment was conducted for three times in an independent manner.

\section{5-Ethynyl-2' -deoxyuridine (EdU) assay}

After the 48-h transfection, TNBC cells after transfection were laid in 96-well plates $\left(1 \times 10^{4}\right.$ cells/well $)$ and labeled using BeyoClick ${ }^{\mathrm{nx}}$ EdU Cell Proliferation Kit (Beyotime, Shanghai, China). Cell nuclei visualization was conducted with the aid of 4',6-diamidino-2-phenylindole (DAPI) staining solution. Eventually, a fluorescence microscope (Olympus, Tokyo, Japan) was used for observing positively labeled cells. The experiment was conducted for three times in an independent manner.

\section{Transwell migration assay}

TNBC cells after transfection were planted in the upper chamber of Transwell chambers (24-well; Corning Incorporated, Corning, NY, USA). Complete culture medium, meanwhile, was filled in the lower chamber which was cultivated with PBS. Twenty-four hours later, cells migrating to the lower chamber were removed with caution by a cotton swab and then fixed in methanol solution for $15 \mathrm{~min}$. Crystal violet was adopted to stain the membranes for $10 \mathrm{~min}$, and the invaded or migrated cells were observed and counted under a microscope (Olympus) at the magnification of $10 \times 10$. The experiment was conducted for three times in an independent manner.

\section{Subcellular separation assay}

Cytoplasmic \& Nuclear RNA Purification Kit was commercially acquired from Norgen Biotek Corp (Thorold, ON, Canada) for conducting subcellular separation assay in TNBC cells according to the instruction from the provider. Cells were treated in cell fractionation buffer to isolate cell cytoplasm. SNHG8 levels in cytoplasmic and nuclear fractions were separately detected by RT-qPCR, using GAPDH and U6 as controls. The experiment was conducted for three times in an independent manner. 
Fluorescence in situ hybridization (FISH) assay

RNA FISH probe specific to SNHG8 was designed and synthesized at RiboBio and utilized as per the instruction. Air-dried cells were incubated with probes in hybridization buffer, and DAPI was adopted to observe nuclei. Images were obtained using a fluorescence microscope (Olympus). The experiment was conducted for three times in an independent manner.

\section{RNA immunoprecipitation (RIP) assay}

Magna RIP ${ }^{\mathrm{mm}}$ RNA-Binding Protein Immunoprecipitation Kit (Millipore, Billerica, MA, USA) was used as per the instruction. Cell lysates and Ago2 antibody (1:50; MA514861; Invitrogen) conjugated on magnetic beads were co-cultured in RIP buffer overnight at $4{ }^{\circ} \mathrm{C}$ through rotation, while IgG antibody (1:500; 31,786; Invitrogen) was utilized as NC. Finally, the RNA precipitates were purified and extracted using the Imprint ${ }^{\circ}$ RNA Immunoprecipitation Kit (RIP-12RXN, Sigma-Aldrich, USA) and RT-qPCR was then applied for relative RNA enrichment examination. The experiment was conducted for three times in an independent manner.

\section{RNA pull down assay}

Pierce Magnetic RNA-Protein Pull-Down Kit (Thermo Scientific, Waltham, MA, USA) was applied for RNA pull down assay according to the standard method from the provider. Protein extracts were collected and incubated with biotinylated miR-335-5p probe, followed by the addition of streptavidin magnetic beads. Then RNAs were eluted down the beads and analyzed using RTqPCR. The experiment was conducted for three times in an independent manner.

\section{Luciferase reporter gene assay}

SNHG8 sequence or the $3^{\prime}$-untranslated region (3'UTR) of PYGO2 with the predicted binding sequence for miR-335-5p was cloned into pmirGLO dualluciferase reporter gene vectors (Promega, Madison, WI, USA), and pmirGLO/SNHG8-WT and pmirGLO/ 3'UTR-WT plasmids were thus obtained. Meanwhile, pmirGLO/SNHG8-Mut and pmirGLO/3'UTR-Mut plasmids were constructed using SNHG8 or the PYGO2 3'UTR sequence with mutant binding sequence for miR335-5p. Luciferase reporter plasmids and other specific plasmids were co-transfected into cells for $48 \mathrm{~h}$, and Dual-Luciferase ${ }^{\bullet}$ Reporter Assay System (Promega) was then applied to examine relative luciferase activity (firefly/Renilla). Assay was performed three times.

\section{Statistical analyses}

All quantitative assays were bio-repeated thrice, and experimental results were displayed as mean \pm standard deviation (SD). A $p$-value less than 0.05 was considered as statistically significant. GraphPad PRISM 6 (GraphPad, La Jolla, CA, USA) was used for analyzing data. Group difference comparison was conducted with Student's $t$ test or one-way/two-way analysis of variance (ANOVA).

\section{Abbreviations}

IncRNA: Long non-coding RNA; ceRNA: Competing endogenous RNA; mRNA: Messenger RNA; miRNA: MicroRNA; shRNA: Short hairpin RNA; UTR: Untranslated region; TNBC: Triple-negative breast cancer; SNHG8: Small nucleolar RNA host gene 8; PYGO2: Pygopus family PHD finger 2; EMT: Epithelial-mesenchymal transition; ATCC: American Type Culture Collection; FBS: Fetal Bovine Serum; FISH: Fluorescence in situ hybridization; RT-qPCR: Real-time quantitative polymerase chain reaction; EdU: 5-ethynyl-2'deoxyuridine; DAPI: 4',6-diamidino-2-phenylindole; SDS-PAGE: Sodium dodecyl sulfate-polyacrylamide gel electrophoresis; PVDF: Polyvinylidene difluoride; RIP: RNA immunoprecipitation; RISC: RNA-induced silencing complex; GAPDH: Glyceraldehyde-3-phosphate dehydrogenase; WT: Wildtype; Mut: Mutant; NC: Negative control; SD: Standard deviation;

ANOVA: Analysis of variance

\section{Acknowledgements}

Thanks a lot for all members of our laboratory.

\section{Authors' contributions}

Jintao Qian designed the study and conceived the experiments with the help of Xinhan Lei, Yue Sun and Lu Zheng. Jia Li and Shuai Zhang conducted the experiments. Lei Zhang and Wanwan Li analyzed data. Jianing Shi and Jintao Qian prepared all the figures, Wenjun Jia and Tong

Tang wrote the paper. The author(s) read and approved the final manuscript.

\section{Funding}

This research is supported by Natural Science Foundation of Anhui Province of China (Grant no.2008085QC112) and Demonstration Project of Grassroots Teaching and Research Section in Anhui Province (Grant no.2018jyssf035).

Availability of data and materials

Not applicable.

\section{Declarations}

Ethics approval and consent to participate Not applicable.

Consent for publication

Not applicable.

\section{Competing interests}

The authors declare that they have no competing interests.

Received: 24 March 2021 Accepted: 28 June 2021

Published online: 06 August 2021

References

1. Anastasiadi Z, Lianos GD, Ignatiadou E, Harissis HV, Mitsis M. Breast cancer in young women: an overview. Updat Surg. 2017;69(3):313-7. https://doi.org/1 0.1007/s13304-017-0424-1.

2. Ghoncheh M, Pournamdar Z, Salehiniya H. Incidence and mortality and epidemiology of breast Cancer in the world. Asian Pac J Cancer Prev. 2016; 17(S3):43-6. https://doi.org/10.7314/APJCP.2016.17.S3.43.

3. Bray F, Ferlay J, Soerjomataram I, Siegel RL, Torre LA, Jemal A. Global cancer statistics 2018: GLOBOCAN estimates of incidence and mortality worldwide for 36 cancers in 185 countries. CA Cancer J Clin. 2018;68(6):394-424. https://doi.org/10.3322/caac.21492.

4. Kumar P, Aggarwal R. An overview of triple-negative breast cancer. Arch Gynecol Obstet. 2016;293(2):247-69. https://doi.org/10.1007/s00404-015-3 859-y.

5. Kaplan HG, Malmgren JA, Atwood MK. Triple-negative breast cancer in the elderly: prognosis and treatment. Breast J. 2017;23(6):630-7. https://doi.org/1 $0.1111 /$ tbj.12813. 
6. Wilusz JE, Sunwoo H, Spector DL. Long noncoding RNAs: functional surprises from the RNA world. Genes Dev. 2009;23(13):1494-504. https://doi. org/10.1101/gad.1800909.

7. Wapinski O, Chang HY. Long noncoding RNAs and human disease. Trends Cell Biol. 2011;21(6):354-61. https://doi.org/10.1016/j.tcb.2011.04.001.

8. Kopp F, Mendell JT. Functional classification and experimental dissection of long noncoding RNAs. Cell. 2018;172(3):393-407. https://doi.org/10.1016/j. cell.2018.01.011.

9. Charles Richard JL, Eichhorn PJA. Platforms for investigating LncRNA functions. SLAS Technol. 2018;23(6):493-506. https://doi.org/10.1177/247263 0318780639

10. Li J, Li L, Yuan H, Huang XW, Xiang T, Dai S. Up-regulated IncRNA GAS5 promotes chemosensitivity and apoptosis of triple-negative breast cancer cells. Cell cycle (Georgetown, Tex). 2019;18(16):1965-75.

11. Wang J, Xi C, Yang X, Lu X, Yu K, Zhang Y, et al. LncRNA WT1-AS inhibits triple-negative breast Cancer cell migration and invasion by Downregulating transforming growth factor $\beta 1$. Cancer Biother Radiopharm. 2019;34(10):671-5. https://doi.org/10.1089/cbr.2019.2925.

12. Ou X, Gao G, Bazhabayi M, Zhang K, Liu F, Xiao X. MALAT1 and BACH1 are prognostic biomarkers for triple-negative breast cancer. J Cancer Res Ther. 2019:15(7):1597-602. https://doi.org/10.4103/jcrt.JCRT_282_19.

13. Zhen Y, Ye Y, Wang H, Xia Z, Wang B, Yi W, et al. Knockdown of SNHG8 repressed the growth, migration, and invasion of colorectal cancer cells by directly sponging with miR-663. Biomed Pharmacotherapy. 2019;116:109000.

14. Dong J, Teng F, Guo W, Yang J, Ding G, Fu Z. IncRNA SNHG8 promotes the tumorigenesis and metastasis by sponging miR-149-5p and predicts tumor recurrence in hepatocellular carcinoma. Cell Physiol Biochem. 2018:51(5): 2262-74. https://doi.org/10.1159/000495871.

15. Chen C, Zhang Z, Li J, Sun Y. SNHG8 is identified as a key regulator in nonsmall-cell lung cancer progression sponging to miR-542-3p by targeting CCND1/CDK6. OncoTargets Ther. 2018;11:6081-90. https://doi.org/10.2147/ OTT.S170482.

16. Yang XZ, Cheng TT, He QJ, Lei ZY, Chi J, Tang Z, et al. LINC01133 as ceRNA inhibits gastric cancer progression by sponging miR-106a-3p to regulate APC expression and the Wnt/ß-catenin pathway. Mol Cancer. 2018;17(1):126.

17. Kong X, Duan Y, Sang Y, Li Y. LncRNA-CDC6 promotes breast cancer progression and function as ceRNA to target CDC6 by sponging microRNA215. J Cell Physiol. 2019;234(6):9105-17.

18. Zheng ZQ, Li ZX, Zhou GQ, Lin L, Zhang LL, Lv JW, et al. Long Noncoding RNA FAM225A Promotes Nasopharyngeal Carcinoma Tumorigenesis and Metastasis by Acting as ceRNA to Sponge miR-590-3p/miR-1275 and Upregulate ITGB3. Cancer Res. 2019;79(18):4612-26.

19. Song Y, Zou L, Li J, Shen ZP, Cai YL, Wu XD. LncRNA SNHG8 promotes the development and chemo-resistance of pancreatic adenocarcinoma. Eur Rev Med Pharmacol Sci. 2018;22(23):8161-8. https://doi.org/10.26355/eurrev_201 812_16508.

20. Song H, Song J, Lu L, Li S. SNHG8 is upregulated in esophageal squamous cell carcinoma and directly sponges microRNA-411 to increase oncogenicity by upregulating KPNA2. OncoTargets Ther. 2019;12:6991-7004. https://doi. org/10.2147/OTT.S214881.

21. Zhang P, Li S, Chen Z, Lu Y, Zhang H. LncRNA SNHG8 promotes proliferation and invasion of gastric cancer cells by targeting the miR-491/ PDGFRA axis. Hum Cell. 2020;33(1):123-30. https://doi.org/10.1007/s13577-01 9-00290-0.

22. Yang $C H$, Zhang $X Y$, Zhou $L N$, Wan $Y$, Song $L L$, Gu WL, et al. LncRNA SNHG8 participates in the development of endometrial carcinoma through regulating c-MET expression by miR-152. Eur Rev Med Pharmacol Sci. 2018; 22(6):1629-37. https://doi.org/10.26355/eurrev_201803_14698.

23. Zhong GB, Jiang $C Q$, Yu XS, Liu ZD, Wang WL, Xu RD. Long noncoding RNA SNHG8 promotes the proliferation of osteosarcoma cells by downregulating miR-542-3p. J Biol Regul Homeost Agents. 2020;34(2):517-24. https://doi. org/10.23812/20-97-61.

24. Fabian MR, Sonenberg N, Filipowicz W. Regulation of mRNA translation and stability by microRNAs. Annu Rev Biochem. 2010;79(1):351-79. https://doi. org/10.1146/annurev-biochem-060308-103103.

25. Luo L, Xia L, Zha B, Zuo C, Deng D, Chen M, et al. miR-335-5p targeting ICAM-1 inhibits invasion and metastasis of thyroid cancer cells. Biomed Pharmacother. 2018;106:983-90

26. Zhang D, Yang N. MiR-335-5p inhibits cell proliferation, migration and invasion in colorectal Cancer through Downregulating LDHB. J BUON. 2019: 24(3):1128-36
27. Chi Y, Wang F, Zhang T, Xu H, Zhang Y, Shan Z, et al. miR-516a-3p inhibits breast cancer cell growth and EMT by blocking the Pygo2/Wnt signalling pathway. J Cell Mol Med. 2019;23(9):6295-307. https://doi.org/10.1111/ jcmm.14515.

\section{Publisher's Note}

Springer Nature remains neutral with regard to jurisdictional claims in published maps and institutional affiliations.

\section{Ready to submit your research? Choose BMC and benefit from:}

- fast, convenient online submission

- thorough peer review by experienced researchers in your field

- rapid publication on acceptance

- support for research data, including large and complex data types

- gold Open Access which fosters wider collaboration and increased citations

- maximum visibility for your research: over $100 \mathrm{M}$ website views per year

At $\mathrm{BMC}$, research is always in progress.

Learn more biomedcentral.com/submissions 